\title{
Reduction of Motion Artifacts and Noise Using Independent Component Analysis in Task-Based Functional MRI for Preoperative Planning in Patients with Brain Tumor
}

\author{
(DE.H. Middlebrooks, (D).J. Frost, DI.S. Tuna, DI.M. Schmalfuss, (D) M. Rahman, and (D) A. Old Crow
}

\begin{abstract}
BACKGROUND AND PURPOSE: Although it is a potentially powerful presurgical tool, fMRI can be fraught with artifacts, leading to interpretive errors, many of which are not fully accounted for in routinely applied correction methods. The purpose of this investigation was to evaluate the effects of data denoising by independent component analysis in patients undergoing preoperative evaluation for glioma resection compared with more routinely applied correction methods such as realignment or motion scrubbing.
\end{abstract}

MATERIALS AND METHODS: Thirty-five functional runs (both motor and language) in 12 consecutive patients with glioma were analyzed retrospectively by double-blind review. Data were processed and compared with the following: 1) realignment alone, 2) motion scrubbing, 3) independent component analysis denoising, and 4) both independent component analysis denoising and motion scrubbing. Primary outcome measures included a change in false-positives, false-negatives, $z$ score, and diagnostic rating.

RESULTS: Independent component analysis denoising reduced false-positives in $63 \%$ of studies versus realignment alone. There was also an increase in the $z$ score in areas of true activation in $71.4 \%$ of studies. Areas of new expected activation (previous false-negatives) were revealed in $34.4 \%$ of cases with independent component analysis denoising versus motion scrubbing or realignment alone. Of studies deemed nondiagnostic with realignment or motion scrubbing alone, $65 \%$ were considered diagnostic after independent component analysis denoising.

CONCLUSIONS: The addition of independent component analysis denoising of fMRI data in preoperative patients with glioma has a significant impact on data quality, resulting in reduced false-positives and an increase in true-positives compared with more commonly applied motion scrubbing or simple realignment methods.

ABBREVIATIONS: BOLD = blood oxygen level-dependent; ICA = independent component analysis; DVARS = root-mean-square of the derivatives of the differentiated time courses of every brain voxel for each acquired volume; $\mathrm{MD}=$ mean displacement; $\mathrm{TC}=$ task correlation

$\mathbf{F}^{\prime}$ unctional MR imaging has become a widely used tool for examining the function of the brain, during both tasks and rest. Currently, the primary clinical impact of fMRI is in presurgical planning, including both epilepsy and tumor applications. The use of preoperative structural and functional images during brain tumor resection has been shown to decrease the duration of the operation, reduce operative complications, and improve patient

Received June 26, 2016; accepted after revision September 7 .

From the Department of Radiology (E.H.M.), University of Alabama at Birmingham, Birmingham, Alabama; Department of Biology (C.J.F.), University of Louisville, Louisville, Kentucky; Medical Imaging Consultants (C.J.F.), Gainesville, Florida; Departments of Radiology (I.S.T., I.M.S., A.O.C.) and Neurosurgery (M.R.), College of Medicine, University of Florida, Gainesville, Florida; and North Florida/South Georgia Veterans Administration (I.M.S.), Gainesville, Florida.

Please address correspondence to Erik H. Middlebrooks, MD, Department of Radiology, University of Alabama at Birmingham, 619 19th St S, JT 3N, Birmingham, AL 35249-6830; e-mail: ehmiddlebrooks@gmail.com

Indicates article with supplemental on-line tables.

Indicates article with supplemental on-line photos.

http://dx.doi.org/10.3174/ajnr.A4996 survival. ${ }^{1,2}$ Unfortunately, fMRI comes with limitations, several of which can lead to impactful interpretive errors.

Blood oxygen level-dependent (BOLD) functional MR imaging relies on the detection of subtle signal changes related to the relationship of oxy- and deoxyhemoglobin as a surrogate marker of neuronal activity. ${ }^{3,4}$ Ideally, signal change should not exist outside that created by perturbations of this balance in oxygenated and deoxygenated blood; unfortunately, numerous other contributions to the signal change confound fMRI data, such as artifacts related to head motion, physiologic noise, and scanner-related noise. ${ }^{5-8}$ In fact, at higher magnetic field strengths, including 3T, the dominant signal in BOLD imaging has been shown to be related to physiologic noise. ${ }^{9}$

Unfortunately, many of the methods routinely used to correct for these artifacts do not fully account for their effect on the data. ${ }^{5,10-12}$ For example, the regularly used "motion correction" algorithms in many fMRI processing programs simply result in spatial realignment of individual voxels for the scan duration. Used in this context, the term "motion correction" is a misnomer. 
It is essential to understand that these typical rigid-body motioncorrection algorithms do not account for significant variance in the data produced by myriad motion-related artifacts. ${ }^{10,11,13}$ Two major culprits are those related to a changing susceptibility profile in the scanner and the effect of the spatial location of spin magnetization saturation, more commonly referred to as spin-history artifacts. ${ }^{11}$ Likewise, numerous other prospective and retrospective methods designed to account for motion-, physiologic-, and scanner-related effects have shown limited improvement. ${ }^{14-18}$

The use of independent component analysis (ICA) for removing the nuisance effects in fMRI data has grown in popularity. ${ }^{19-21}$ Fundamentally, ICA separates a mixture of signals in a dataset into its individual signal components. ICA is commonly explained with the analogy of a cocktail party, in which the recorded, indistinct sound from numerous conversations can be deconstructed into individual voices. ICA denoising has been shown to increase statistical significance and sensitivity, resulting in fewer false-positives and false-negatives. ${ }^{22}$ The goal of our study was to evaluate the role of ICA denoising on fMRI data of patients undergoing preoperative planning for brain tumor resection.

\section{MATERIALS AND METHODS}

The requirement for informed consent was waived in this Health Insurance Portability and Accountability Act-compliant retrospective study, which was approved by the University of Florida institutional review board. Thirty-five runs from 12 consecutive patients undergoing preoperative planning for glioma resection were retrospectively reviewed. Various language (semantic decision [6/35], sentence completion [3/35], word generation [5/35], picture naming [3/35]) and motor (finger [6/35], foot [4/35], tongue [2/35], face [6/35]) paradigms were used. See On-line Table 1 for details of the task designs. All patients were trained on the tasks by the administering physician (E.H.M.).

\section{Image Acquisition}

MR imaging data were acquired on a 3T Verio scanner (Siemens, Erlangen, Germany) with a 12-channel head coil. In an attempt to minimize head motion, the head was tightly packed with moldable foam. The importance of reducing head motion was explained to patients. A volumetric T1-weighted MPRAGE sequence with a voxel size of $1 \times 1 \times 1 \mathrm{~mm}, \mathrm{TR}=2530 \mathrm{~ms}, \mathrm{TE}=3.5$ $\mathrm{ms}, \mathrm{TI}=1100 \mathrm{~ms}$, and flip angle $=7^{\circ}$ was obtained. BOLD-EPI images used a $3.5 \times 3.5 \times 3.5 \mathrm{~mm}$ voxel size with a $0.7-\mathrm{mm}$ intersection gap, $\mathrm{TR}=2000 \mathrm{~ms}, \mathrm{TE}=30 \mathrm{~ms}$, and flip angle $=78^{\circ}$, with an oblique axial interleaved acquisition. All patients were monitored and scanned by the administering physician (E.H.M.).

\section{Data Processing}

Echo-planar images were processed with the fMRIB Software Library (FSL, Version 5.0; http://www.fmrib.ox.ac.uk/fsl). ${ }^{23}$ Preprocessing of all datasets included realignment (Motion Correction using FMRIB's Linear Image Registration Tool [MCFLIRT]; http://fsl.fmrib.ox.ac.uk/fsl/fslwiki/MCFLIRT), ${ }^{24}$ section-timing correction, and spatial smoothing (5-mm full width at half maximum). The preprocessed data were then processed using 4 differing methods. First, the preprocessed, realigned data were processed without any further manipulation. Second, motion scrubbing was performed by regressing individual volumes that exceeded a strict threshold for excessive section-signal variation based primarily on mean displacement and root-mean-square of the derivatives of the differentiated time courses of every brain voxel for each acquired volume (DVARS), as described in Power et al. ${ }^{25}$ Third, ICA analysis was performed (Multivariate Exploratory Linear Optimized Decomposition into Independent Components [MELODIC]; http://fsl.fmrib.ox.ac.uk/fsl/fslwiki/ MELODIC), and nuisance components were manually identified by visual inspection of components by an experienced ICA user (E.H.M.) and were removed by using the fsl_regfilt command. The methodology of characterizing components as noise- or taskrelated signal is based on the methodology used in the excellent review presented in Kelly et al. ${ }^{26}$ Last, both ICA denoising and motion scrubbing were performed, as previously described.

Data were analyzed using the FSL General Linear Model (http://fsl.fmrib.ox.ac.uk/fsl/fslwiki/GLM) with cluster thresholding in FMRIB Expert Analysis Tool (FEAT; http://fsl.fmrib.ox. ac.uk/fsl/fslwiki/FEAT). Identical threshold levels were applied to all 4 processing methods to determine the effects of the 4 processing pipelines on statistical significance. Processed EPI data were then linearly registered to the T1-weighted structural images (FMRIB Linear Image Registration [FLIRT]; http://www.fmrib. ox.ac.uk). Data for all 4 processing methods were overlaid to detect differences in each methodology. Two neuroradiologists with functional imaging experience (E.H.M., I.S.T.) independently and blindly evaluated the overlaid data to assess the presence or absence of expected activation based on the task, amount of noise, and change in statistical significance $(z$ score variance) of the activated areas and whether any or all of the processing methods were considered diagnostic. Diagnostic studies were defined as showing all expected areas of activation for the given task regardless of tumor location, to minimize the subjectivity of the meaning of diagnostic versus nondiagnostic. The subjective measures of noise, new real areas of activation, and diagnostic ability for each method resulted in 560 individual binary data points, which were entered in to an assessment of reader agreement. Disagreements were settled by a third blinded reader (I.M.S.).

\section{Data Analysis}

We extensively evaluated image quality and tabulated 3 primary motion parameters: mean displacement (MD), task correlation (TC), and DVARS. For MD, each functional run was placed in 1 of 3 categories from none/mild, moderate, to severe, as follows: $<1$-mm MD throughout the study, 1 - to $2-\mathrm{mm}$ MD in $\leq 4$ spikes, or any $\mathrm{MD}$ of $>2 \mathrm{~mm}$ or $>1 \mathrm{~mm}$ for $>4$ spikes. Likewise, DVARS was categorized as the following: no spikes of $>5 \%$ signal change from volume to volume, $1-5$ spikes of $>5 \%$, or $>5$ spikes of $>5 \%$. TC was categorized as $r<0.05,0.05 \leq r<0.2$, or $r \geq 0.2$.

Due to the ordinal and nominal nature of the data, contingency analysis was used with motion parameters (ordinal) as independent measurements to evaluate their relationships to the nominal, binary (yes/no) response outcome measures. The CochranArmitage exact test for trend was appropriate to capture the power of the ordinal motion parameter measurements to assess statistical significance; the Fisher exact test was used when the motion parameter data were collapsed into binary variables 


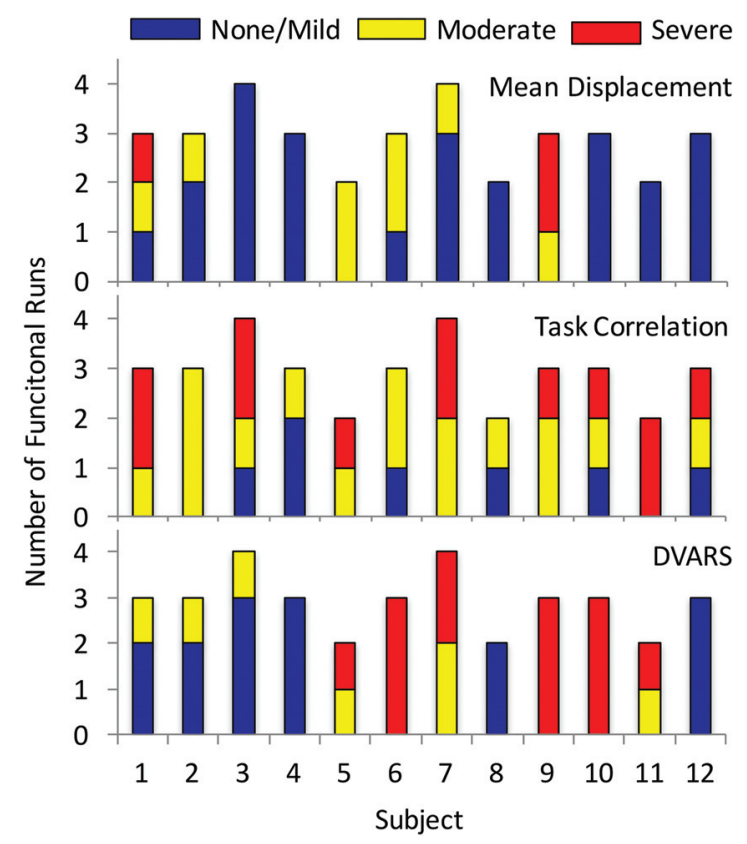

FIG 1. Distribution of motion parameters in each individual subject showing the number of functional runs characterized as having a none/mild, moderate, or severe rating in each of the 3 recorded parameters (DVARS, task-correlated motion, and MD).

(eg, assessing ICA-salvaged diagnostic studies). $\chi^{2}$ was used to assess expected-versus-observed effects among the 12 subjects, including subject-level variation of the motion parameter severity and comparing the number of diagnostic-versus-nondiagnostic scans when realignment alone, motion scrubbing, ICA, or both ICA and motion scrubbing was applied.

\section{RESULTS}

The 2 readers' independent evaluations agreed in 97\% (546/560) of individual recorded data points; only 14 evaluations required a tie-breaker with a blinded third reader (On-line Table 2).

\section{Motion Parameters}

The individual motion parameters are illustrated in Fig 1. A severe rating was present in 1 of the 3 motion parameters in $60 \%(21 / 35)$ of studies and in $>1$ motion parameter in 17\% (6/35) of studies. MD and DVARS were patient-specific: Half of patients had none/ mild MD $\left(\chi^{2}=35.5, P=.034\right)$, while half of patients accounted for nearly all $(17 / 20)$ moderate or severe DVARS $\left(\chi^{2}=39.3, P=\right.$ $.013)$. In contrast, task-correlated motion of $>0.05$ was observed in at least 1 paradigm in all 12 patients, with severe task-correlated motion distributed across 8 patients $\left(\chi^{2}=20.6, P=.547\right)$. While there were no statistical differences in motion parameters between language and motor studies, motor tasks accounted for 8 of the 12 severe TC motion studies (On-line Fig 1).

\section{fMRI Statistical Analyses}

ICA denoising resulted in demonstrable improvement in overall fMRI quality. ICA denoising decreased the level of noise (falsepositives) in the activation maps in 63\% (22/35) of cases compared with realignment alone. A reduction in noise after ICA denoising was not correlated with any individual motion parameter or combination thereof. ICA also improved the statistical signifi-

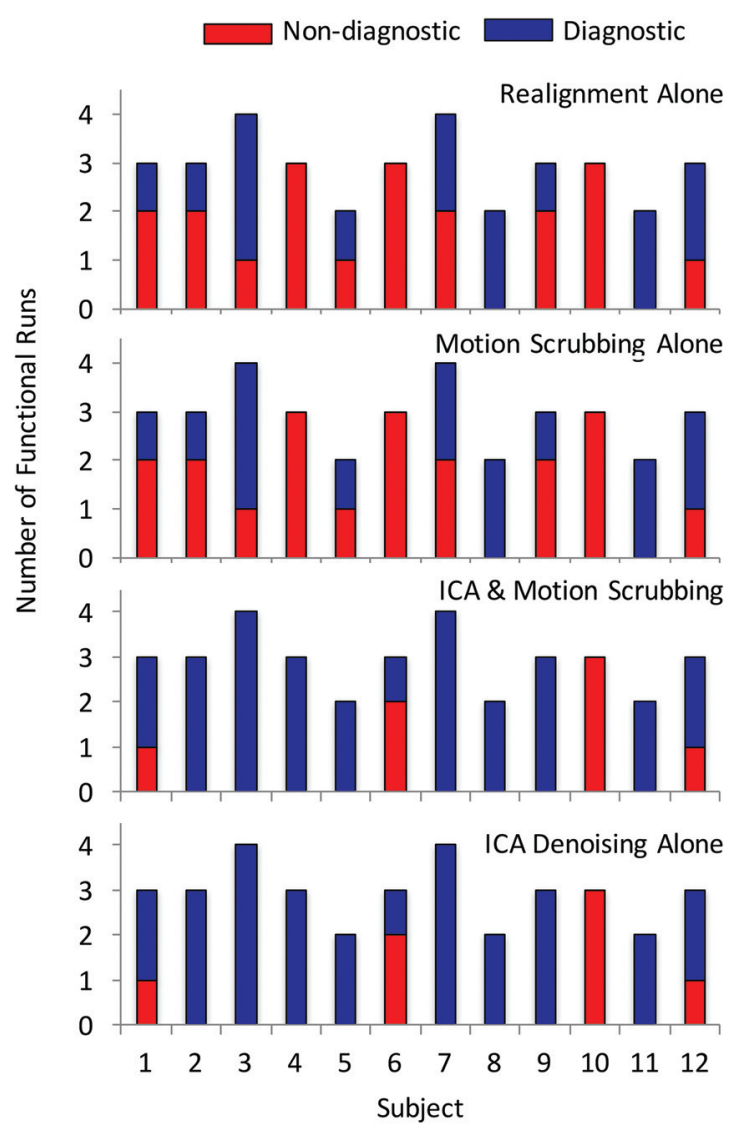

FIG 2. Distribution of diagnostic and nondiagnostic scans for each correction method per subject.

cance in regions of expected activation in $71.4 \%(25 / 35)$ of cases compared with realignment or motion scrubbing alone. ICA improved fMRI statistics, as assessed by an increase in the $z$ score in areas of expected activation, in almost all scans with high $\mathrm{MD}$ (7/8 and $3 / 3$ cases of moderate and severe $\mathrm{MD}$, respectively; $z=-1.75$, $P=.040)$. Additionally, ICA improved fMRI statistics with moderate or high TC $(z=-1.80, P=.036)$ and when MD and TC were considered together $(z=-1.92, P=.049)$. New expected areas of activation (previous false-negatives) were present only after ICA denoising in $34.3 \%$ (12/35) of the datasets and were not correlated with any motion parameter. Motion scrubbing alone did not reveal new areas of expected activation (previous falsenegatives) compared with realignment alone or ICA. The combination of ICA and motion scrubbing showed no difference from ICA alone.

\section{Diagnostic Quality}

Most important, ICA denoising improved the diagnostic value of the fMRI studies (Fig 2). After realignment alone, 10 of the 12 patients had at least 1 nondiagnostic scan $\left(\chi^{2}=14.9, P=.186\right.$; Fig 2 , upper graph), with 3 patients having all nondiagnostic-quality scans. In all, 57\% (20/35) of scans were deemed nondiagnostic when corrected with realignment only. ICA denoising rendered diagnostic $65 \%(13 / 20)$ of the previously nondiagnostic scans, increasing the overall percentage of diagnostic scans from $42.9 \%$ to $80.0 \%\left(\chi^{2}=6.3, P<.001\right)$. Moreover, ICA denoising improved the diagnostic outcome for 11 of 12 patients, with 8 pa- 
tients having all scans of diagnostic quality $\left(\chi^{2}=22.5, P=.021\right.$; Fig 2, lower graph). Notably, all 7 scans (in 4 patients) that remained nondiagnostic after ICA denoising contained severe TC and/or DVARS ( $z=2.76, P=.004)$. In contrast, motion scrubbing failed to improve any of these scans to the point of being diagnostic (Fig 2, second graph), which is in line with the failure of motion scrubbing to reveal any new areas of activation compared with motion correction alone.

Motion scrubbing alone increased the statistical significance in expected areas of activation in 20\% (7/35) of the scans compared with realignment alone but only showed improved statistical significance versus ICA denoising in 1 case (with low MD, severe TC, and moderate DVARS). Motion scrubbing had the greatest effect in those scans with moderate-severe ratings in TC alone $(z=-1.75, P=.040)$ or to a lesser extent when MD and TC were considered together $(z=-1.67, P=.054)$. The addition of motion scrubbing to ICA denoising did not show any improvement over ICA denoising alone.

\section{DISCUSSION}

Based on the previously reported success of ICA in improving data quality in both task-based and resting-state fMRI studies, our goal was to investigate its utility in preoperative planning for patients undergoing resection for gliomas. We found improvement in statistical significance and decreased noise, resulting in fewer false-positives and, perhaps more important, an increase in truepositives, similar to findings in studies in patients without tumors. ${ }^{20,27,28}$ We also found that a proportion of studies, otherwise nondiagnostic when processed with traditional methods, were diagnostic after ICA denoising.

As previously discussed, underlying motion-related artifacts, physiologic noise, and scanner-related noise, which are ubiquitous in fMRI, have serious detrimental effects on fMRI interpretation. The first of these, subject motion, is becoming an increasingly recognized cause of fMRI misinterpretation. For example, a prominent theory in autism that touted decreased long-range connections was eventually shown to be related to increased head motion in the autistic group relative to controls. ${ }^{12,29}$ For any user of fMRI, it is essential to have a thorough understanding of motion and related artifacts. An important tenet of high-quality fMRI is homogeneity of the B0 magnetic field. When an object is introduced into the bore of the scanner, the field becomes distorted and is corrected for with a shimming procedure. However, if the object moves once in the bore, this shim effect is lost and the susceptibility profile of the object changes. This change can result in drastic changes in signal intensity and geometric distortion that cannot be corrected by typical realignment procedures. ${ }^{11,13}$ Shifts as small as $2^{\circ}$ can potentially create large areas of false-positive activation regardless of intensive thresholding. ${ }^{13}$

A second major consideration regarding patient motion in fMRI is signal change as a result of spin-history artifacts. Given the typical short TRs in fMRI, the brain achieves a steady-state of incomplete longitudinal magnetization recovery after a few TRs. This recovery is slightly out of phase in any individual section. Once the head moves, these voxels will be shifted into a different plane, resulting in either a lower- or higher-than-expected amount of recovery in sections obtained prior or subsequent to the reference section, respectively. This unexpected change in magnetization recovery can have a substantial effect on signal intensity. ${ }^{11}$ While abrupt motion with a large amount of displacement can have noticeable effects on the signal intensity, minimal amounts of motion ( $<1$-mm displacement) can have a substantial impact on the data if the motion is frequent. Because this movement is low-amplitude, one cannot expect to see significant outliers in mean signal intensity across volumes. Spin-history artifacts are best assessed by DVARS, which assesses the volume-tovolume signal change. ${ }^{25}$ In our data, we found this frequent, lowamplitude motion $(<1 \mathrm{~mm})$ effect to be the most common source of nondiagnostic studies.

Third, when signal fluctuations from artifacts, such as the previously mentioned spin-history artifacts and susceptibility changes, create a time-series similar to the experimental design, commonly referred to as "task-correlated motion," the artifactual component is generally inseparable from task-related signal change. ${ }^{8,28,30}$ Our results show that task-correlated motion has a strong prevalence in nondiagnostic studies, including studies that are not even improved by ICA denoising. Although neurovascular uncoupling could potentially be the source of these nondiagnostic studies, the prevalence of task-correlated motion suggests that this may also be an etiology. Given the impact of TC on study outcomes, the assessment of this parameter should be a routine part of fMRI data quality analysis.

Physiologic noise is also a major source of variance in BOLD fMRI data and is primarily related to fluctuations in basal metabolism, cardiac and respiratory effects, and subtle motion effects from brain pulsation. In fact, it has been shown that physiologic noise at a higher field strength (3T) is the dominant source of noise in fMRI and even counteracts much of the gain in signalto-noise ratio and contrast-to-noise ratio when moving to higher magnetic field strengths. ${ }^{9,31}$ Due to the typically short TRs used in fMRI, the frequency of these artifacts results in their aliasing into the task-related signal. ${ }^{7}$ Because this noise is structured, nonwhite noise, it does not meet the statistical assumption that errors are independent and identically distributed; therefore, it will have a significant impact on most statistical modeling. ${ }^{32}$ Similarly, scanner-related noise, such as thermal noise, drift, and imperfections in the coil, radiofrequency, gradient, and shim subsystems and various other minor contributors, produces similar effects. ${ }^{9}$ Our study revealed a large decrease in false-positives with use of ICA versus other methods; however, the ICA decrease in false-positives did not correlate with any motion parameters. This finding could suggest that removal of physiologic and scanner noise is also a major contributor to improvement seen with ICA versus other methods.

Many attempts have been made to reduce artifacts in fMRI; however, they remain extremely problematic. There are generally 2 types of artifact-correction systems, hypothesis-driven and data-driven. Hypothesis-driven systems include prospective motion-correction systems, such as the use of navigator sequences, ${ }^{33,34}$ optical motion tracking, ${ }^{16,18,35}$ or methods of measuring physiologic parameters during the scan (ie, heart rate, respiratory rate, and so forth) that are then used to apply filters to the data or used as nuisance regressors. ${ }^{7,14}$ Unfortunately, these methods have been difficult to implement due to inaccurate cardiac or respiratory peak detection, variability in results with differing TRs, lag in the motion data, and 


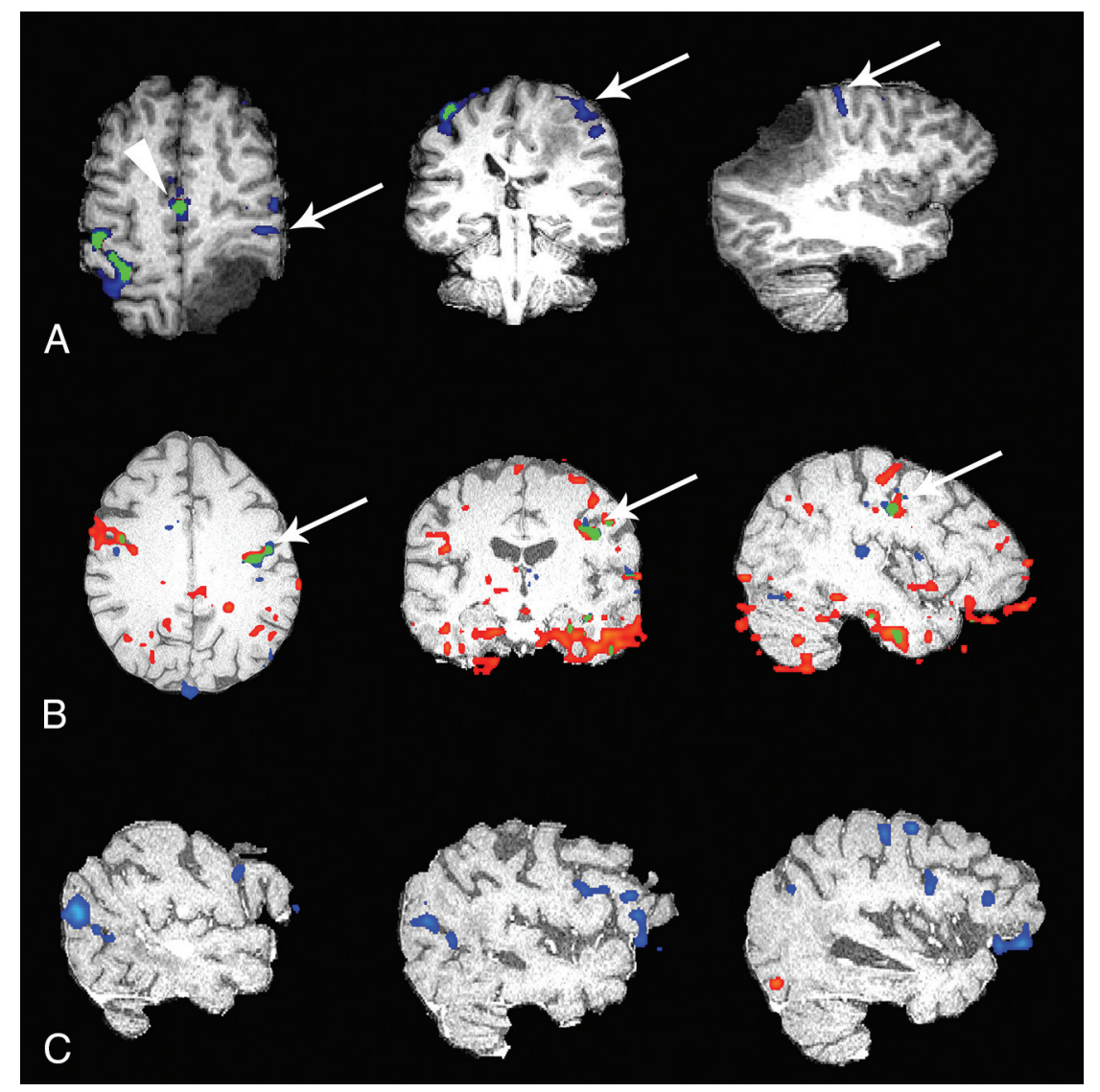

FIG 3. Processed fMRI data from 3 different subjects. Each case shows ICA-denoised data in blue, motion-scrubbed data in red, and overlapping areas of ICA-denoised data and motion-scrubbed data in green. The first case $(A)$ is a motor finger task in a patient with a left parietal glioma. The primary motor cortex for the right finger (arrow) is only seen after ICA denoising. Likewise, the supplementary motor area (arrowhead) shows a slight increase in statistical significance. The second subject $(B)$ is undergoing a motor face task with severe task-correlated motion and severe DVARS showing no major change in the primary motor face cortex (arrow); however, the number of motion-related false-positives (noise) is markedly reduced. The third case $(C)$ is a semantic decision task in which no meaningful activation is present on the motion-scrubbed data. Expected areas of activation in the anterior and posterior language areas are clearly present after ICA denoising.

increased radiofrequency pulses resulting in decreased temporal resolution. Furthermore, no single method accounts for all sources of noise present in the data. ${ }^{5,7,32,36}$

Data-driven approaches, such as removal or regression of volumes with signal changes above a threshold (motion scrubbing) or regression of motion parameters, do not account for all sources of artifacts and have limited effect. ${ }^{5,25}$ The limited effect on quality improvement is evident when volumes are discarded on the basis of motion parameters alone, because motion artifacts can have serious detrimental effects, even with minor head motion $(<1 \mathrm{~mm}){ }^{8}$ Thus, more thorough parameters are needed when using motion scrubbing, such as DVARS or frame-wise displacement, which can detect very subtle artifactual changes in signal. ${ }^{25}$ Furthermore, motion scrubbing results in decreased statistical power, particularly in data with numerous motion-affected volumes, while images with detrimental artifacts that do not meet the threshold for rejection still exist. Regression of the motion parameters can also have deleterious effects if the motion is correlated with the task and therefore must be used with extreme caution in task-based experiments. ${ }^{6}$ Even with these considerations, the overall improvement gained by these methods is minimal. ${ }^{5,12,25}$ This outcome is also likely due, in part, to the lack of accounting for physiologic and scanner-related noise.

There has been an increasing interest in the use of ICA to denoise fMRI data to improve the sensitivity and specificity of results. The methodology behind ICA is beyond the scope of this article (see Stone $^{37}$ for an excellent introduction), but in short, ICA deconstructs an fMRI time-series into unique components (each having a spatial map and the corresponding time course), which are maximally spatially independent. ${ }^{19,22,28,37}$ These components can then be classified as either artifactual/noise versus taskrelated activity (see Kelly et al, ${ }^{26}$ for an excellent review). In addition to manual identification, numerous methods of automated classification have been described, primarily by using machinelearning algorithms, but a discussion of these methods is beyond the scope of this article. ${ }^{38-43}$ The artifactual components consist of a variety of physiologic processes, motion-related artifacts, scannerrelated noise, aliasing, and so forth. Once identified, these artifactual components can be removed from the dataset before statistical analysis, such as with the General Linear Model. The improved statistical modeling results in increased sensitivity and specificity, with reduction of false-positives and increased true-positives (Fig 3). ${ }^{20,27,28}$

Previous studies evaluating the use of ICA in task-based presurgical fMRI have yielded mixed results. ${ }^{21,44,45}$ While these studies were the first to explore ICA in the presurgical setting, they relied on the extraction of a single component from the ICA analysis results that best modeled the task. This approach can be problematic because taskrelated activation can often be distributed across multiple components. ${ }^{46}$ An approach rendering the most accurate and reliable results is to remove those components reflecting structured or random noise during preprocessing and then to process the denoised dataset with statistical modeling. Additionally, these studies were performed at $1.5 \mathrm{~T}$, in which physiologic noise and susceptibility-related changes are substantially lower than at 3T, so the results may not be directly applicable to higher field strengths.

The results of this study highlight the benefits of ICA denoising in presurgical mapping for tumor resection, even at higher field strengths. The reduction in false-positives related to these nuisance variables also reduces the number of surgical false-positives. Furthermore, this reduction has the effect of providing the surgeon with "cleaner" activation maps. Additionally, we have demonstrated that in some cases, true-positive activation was only identified with the use of ICA denoising, the lack of which could have had detrimental effects on the operation (Fig 4). The 


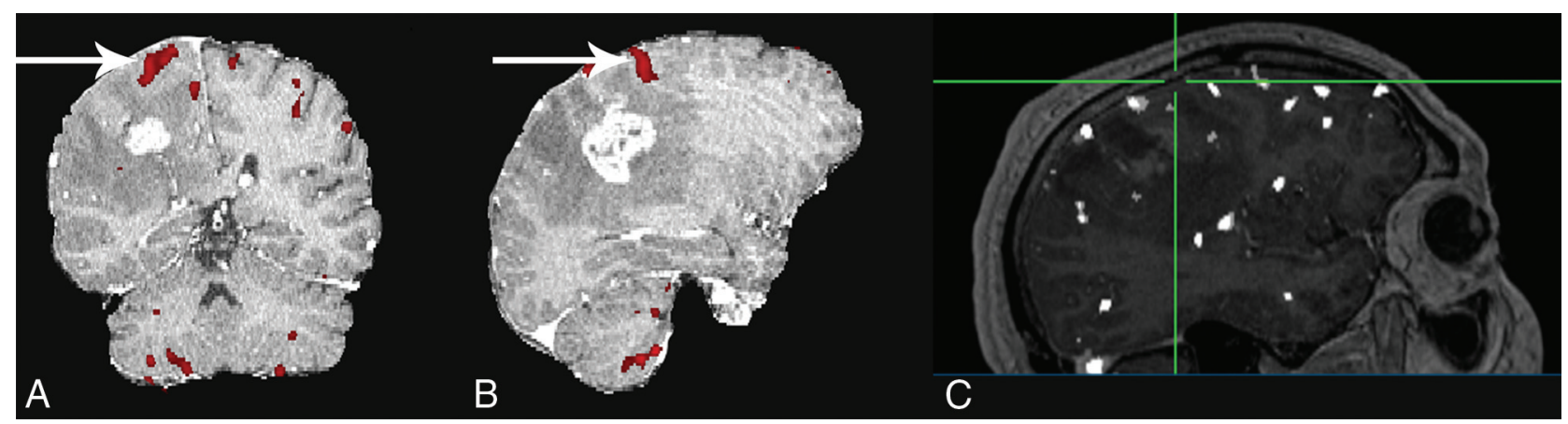

FIG 4. $A$ and $B$, Images for a subject undergoing a motor finger task showing areas in which the $z$ score increased (red) after ICA denoising compared with motion scrubbing. The arrow illustrates increased statistical significance in the left finger primary motor cortex adjacent to the tumor. The location of the primary motor cortex for the left finger was confirmed surgically and is shown on the intraoperative map (C), where the crosshairs correspond to primary finger motor cortex. The white "blobs" in C show the thresholded maps for the realignment-only data where there is no activation in the area of the primary motor cortex.
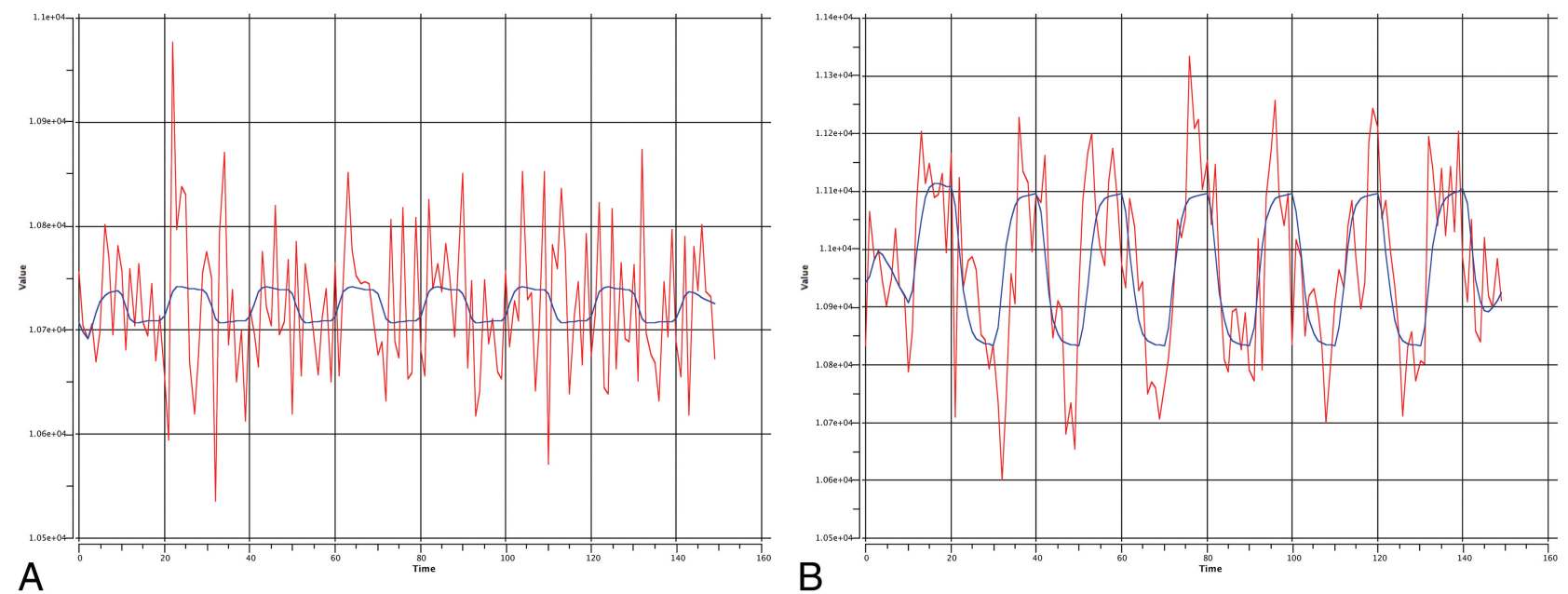

FIG 5. Signal intensity with time from 1 voxel is shown before $(A)$ and after $(B)$ ICA denoising with the expected hemodynamic response in blue. One can appreciate the amount of overlapping noise from a variety of artifacts resulting in a poor fit with the ideal curve. These noise effects are largely removed after ICA denoising, and the subsequent denoised time course shows a substantially improved fit with the task design.

appearance of otherwise undetected activation is likely due to improved statistical significance achieved by the removal of overlapping nuisance signal or artifacts in the area of true activation (Fig 5). This effect was most dramatic in patients with a higher incidence of motion-induced artifacts. However, when motion was correlated with the task, the motion-induced signal changes were generally indistinguishable from the task itself and any improvement in data quality with ICA denoising, as well as motion scrubbing, was often negligible. Last, we have shown an overall improvement in statistical significance of activated voxels with the use of ICA denoising.

Notable limitations of the current study include its retrospective nature. Because limited evidence exists on the effects of ICA denoising in the surgical setting, we do not believe that the prospective use of this technique is yet warranted. In this retrospective design, a strong inherent bias is present when assessing whether the results would alter patient treatment or decisionmaking, and as such, this assessment was not included in the analysis. Additionally, precise intraoperative mapping data were not collected in a structured fashion; this omission limits the ability to directly correlate with surgical data in all patients. Likewise, an inherent limitation of many surgical mapping studies is the inability to test all activation sites from limited craniotomies. To address these limitations, we relied on a double-blind expert review of activation maps to assess activation in expected areas on the basis of the task administered. We believe these limitations do not detract from the objective of this study in identifying data-quality improvement with ICA denoising relative to other standard methods of fMRI data denoising. Future prospective studies should be aimed at evaluating the impact on decision-making and patient outcomes.

\section{CONCLUSIONS}

The addition of ICA denoising of fMRI data in preoperative patients with glioma has a significant impact in data quality, resulting in reduced false-positives and an increase in true-positives compared with more commonly applied motion scrubbing or simple realignment methods.

\section{REFERENCES}

1. Pillai JJ. The evolution of clinical functional imaging during the past 2 decades and its current impact on neurosurgical planning. AJNR Am J Neuroradiol 2010;31:219-25 CrossRef Medline

2. Krings T, Reinges MH, Erberich S, et al. Functional MRI for presurgical planning: problems, artefacts, and solution strategies. J Neurol Neurosurg Psychiatry 2001;70:749-60 CrossRef Medline

AJNR Am J Neuroradiol 38:336-42 Feb 2017 www.ajnr.org 
3. Ogawa S, Lee TM, Kay AR, et al. Brain magnetic resonance imaging with contrast dependent on blood oxygenation. Proc Natl Acad Sci U S A 1990;87:9868-72 CrossRef Medline

4. Ogawa S, Menon RS, Tank DW, et al. Functional brain mapping by blood oxygenation level-dependent contrast magnetic resonance imaging: a comparison of signal characteristics with a biophysical model. Biophys J 1993;64:803-12 CrossRef Medline

5. Xu Y, Tong Y, Liu S, et al. Denoising the speaking brain: toward a robust technique for correcting artifact-contaminated fMRI data under severe motion. Neuroimage 2014;103:33-47 CrossRef Medline

6. Johnstone T, Ores Walsh KS, Greischar LL, et al. Motion correction and the use of motion covariates in multiple-subject fMRI analysis. Hum Brain Mapp 2006;27:779-88 CrossRef Medline

7. Biswal B, DeYoe EA, Hyde JS. Reduction of physiological fluctuations in fMRI using digital filters. Magn Reson Med 1996;35:107-13 CrossRef Medline

8. Field AS, Yen YF, Burdette JH, et al. False cerebral activation on BOLD functional MR images: study of low-amplitude motion weakly correlated to stimulus. AJNR Am J Neuroradiol 2000;21: 1388-96 Medline

9. Krüger G, Glover GH. Physiological noise in oxygenation-sensitive magnetic resonance imaging. Magn Reson Med 2001;46:631-37 CrossRef Medline

10. Wilke M. Isolated assessment of translation or rotation severely underestimates the effects of subject motion in fMRI data. PLoS One 2014;9:e106498 CrossRef Medline

11. Friston KJ, Williams S, Howard R, et al. Movement-related effects in fMRI time-series. Magn Reson Med 1996;35:346-55 CrossRef Medline

12. Power JD, Barnes KA, Snyder AZ, et al. Spurious but systematic correlations in functional connectivity MRI networks arise from subject motion. Neuroimage 2012;59:2142-54 CrossRef Medline

13. Wu DH, Lewin JS, Duerk JL. Inadequacy of motion correction algorithms in functional MRI: role of susceptibility-induced artifacts. J Magn Reson Imaging 1997;7:365-70 CrossRef Medline

14. Glover GH, Li TQ, Ress D. Image-based method for retrospective correction of physiological motion effects in fMRI: RETROICOR. Magn Reson Med 2000;44:162-67 Medline

15. Goto M, Abe O, Miyati T, et al. Head motion and correction methods in resting-state functional MRI. Magn Reson Med Sci 2016;15: 178-86 CrossRef Medline

16. Todd N, Josephs O, Callaghan MF, et al. Prospective motion correction of 3D echo-planar imaging data for functional MRI using optical tracking. Neuroimage 2015;113:1-12 CrossRef Medline

17. Muraskin J, Ooi MB, Goldman RI, et al. Prospective active marker motion correction improves statistical power in BOLD fMRI. Neuroimage 2013;68:154-61 CrossRef Medline

18. Schulz J, Siegert T, Bazin PL, et al. Prospective slice-by-slice motion correction reduces false positive activations in $\mathrm{AMRI}$ with task-correlated motion. Neuroimage 2014;84:124-32 CrossRef Medline

19. McKeown MJ, Makeig S, Brown GG, et al. Analysis of fMRI data by blind separation into independent spatial components. Hum Brain Mapp 1998;6:160-88 Medline

20. Biswal BB, Ulmer JL. Blind source separation of multiple signal sources of fMRI data sets using independent component analysis. J Comput Assist Tomogr 1999;23:265-71 CrossRef Medline

21. Quigley MA, Haughton VM, Carew J, et al. Comparison of independent component analysis and conventional hypothesis-driven analysis for clinical functional MR image processing. AJNR Am J Neuroradiol 2002;23:49-58 Medline

22. Thomas CG, Harshman RA, Menon RS. Noise reduction in BOLDbased fMRI using component analysis. Neuroimage 2002;17:1521-37 CrossRef Medline

23. Smith SM, Jenkinson M, Woolrich MW, et al. Advances in functional and structural MR image analysis and implementation as FSL. Neuroimage 2004;23(suppl 1):S208-19 CrossRef Medline

24. Jenkinson M, Bannister $\mathrm{P}, \mathrm{Brady} \mathrm{M}$, et al. Improved optimization for the robust and accurate linear registration and motion correction of brain images. Neuroimage 2002;17:825-41 CrossRef Medline
25. Power JD, Mitra A, Laumann TO, et al. Methods to detect, characterize, and remove motion artifact in resting state fMRI. Neuroimage 2014;84:320-41 CrossRef Medline

26. Kelly RE Jr, Alexopoulos GS, Wang Z, et al. Visual inspection of independent components: defining a procedure for artifact removal from fMRI data. J Neurosci Methods 2010;189:233-45 CrossRef Medline

27. McKeown MJ, Hansen LK, Sejnowsk TJ. Independent component analysis of functional MRI: what is signal and what is noise? Curr Opin Neurobiol 2003;13:620-29 CrossRef Medline

28. Kochiyama T, Morita T, Okada T, et al. Removing the effects of task-related motion using independent-component analysis. $\mathrm{Neu}$ roimage 2005;25:802-14 CrossRef Medline

29. Power JD, Fair DA, Schlaggar BL, et al. The development of human functional brain networks. Neuron 2010;67:735-48 CrossRef Medline

30. Desmond JE, Atlas SW. Task-correlated head movement in fMR imaging: false activations can contaminate results despite motion correction. AJNR Am J Neuroradiol 2000;21:1370-71 Medline

31. Krüger G, Kastrup A, Glover GH. Neuroimaging at $1.5 \mathrm{~T}$ and $3.0 \mathrm{~T}$ : comparison of oxygenation-sensitive magnetic resonance imaging. Magn Reson Med 2001;45:595-604 CrossRef Medline

32. Lund TE, Madsen KH, Sidaros K, et al. Non-whitenoise in fMRI: does modelling have an impact? Neuroimage 2006;29:54-66 CrossRef Medline

33. White N, Roddey C, Shankaranarayanan A, et al. PROMO: real-time prospective motion correction in MRI using image-based tracking. Magn Reson Med 2010;63:91-105 CrossRef Medline

34. Ward HA, Riederer SJ, Grimm RC, et al. Prospective multiaxial motion correction for fMRI. Magn Reson Med 2000;43:459-69 Medline

35. Rotenberg D, Chiew M, Ranieri S, et al. Real-time correction by optical tracking with integrated geometric distortion correction for reducing motion artifacts in functional MRI. Magn Reson Med 2013; 69:734-48 CrossRef Medline

36. Dold C, Zaitsev M, Speck O, et al. Advantages and limitations of prospective head motion compensation for MRI using an optical motion tracking device. Acad Radiol 2006;13:1093-103 CrossRef Medline

37. Stone JV. Independent component analysis: an introduction. Trends Cogn Sci 2002;6:59-64 CrossRef Medline

38. Bhaganagarapu K, Jackson GD, Abbott DF. An automated method for identifying artifact in independent component analysis of resting-state FMRI. Front Hum Neurosci 2013;7:343 CrossRef Medline

39. Sochat V, Supekar K, Bustillo J, et al. A robust classifier to distinguish noise from $\mathrm{fMRI}$ independent components. PLoS One 2014;9: e95493 CrossRef Medline

40. Salimi-Khorshidi G, Douaud G, Beckmann CF, et al. Automatic denoising of functional MRI data: combining independent component analysis and hierarchical fusion of classifiers. Neuroimage 2014;90:449-68 CrossRef Medline

41. Tohka J, Foerde K, Aron AR, et al. Automatic independent component labeling for artifact removal in fMRI. Neuroimage 2008;39: 1227-45 CrossRef Medline

42. De Martino F, Gentile F, Esposito F, et al. Classification of fMRI independent components using IC-fingerprints and support vector machine classifiers. Neuroimage 2007;34:177-94 CrossRef Medline

43. Pruim RH, Mennes M, van Rooij D, et al. ICA-AROMA: A robust ICA-based strategy for removing motion artifacts from fMRI data. Neuroimage 2015;112:267-77 CrossRef Medline

44. Moritz CH, Haughton VM, Cordes D, et al. Whole-brain functional MR imaging activation from a finger-tapping task examined with independent component analysis. AJNR Am J Neuroradiol 2000;21: 1629-35 Medline

45. Caulo M, Esposito R, Mantini D, et al. Comparison of hypothesisand a novel hybrid data/hypothesis-driven method of functional MR imaging analysis in patients with brain gliomas. AJNR Am J Neuroradiol 2011;32:1056-64 CrossRef Medline

46. McKeown MJ. Detection of consistently task-related activations in fMRI data with hybrid independent component analysis. Neuroimage 2000;11:24-35 CrossRef Medline 